\title{
Conference report: inaugural Pharmacogenomics Access \& Reimbursement Symposium
}

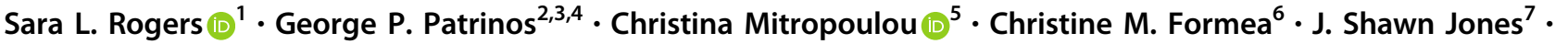 \\ Benjamin G. Brown ${ }^{1}$
}

Received: 26 January 2021 / Revised: 23 March 2021 / Accepted: 23 April 2021 / Published online: 17 June 2021

(c) The Author(s), under exclusive licence to Springer Nature Limited 2021

The Pharmacogenomics Access \& Reimbursement Symposium, a landmark event presented by the Golden Helix Foundation and the Pharmacogenomics Access \& Reimbursement Coalition (PARC), was a one-day interactive meeting comprised of plenary keynotes from thought leaders across health care that focused on value-based strategies to improve patient access to personalized medicine. Stakeholders including patients, healthcare providers, industry, government agencies, payer organizations, health systems and health policy organizations convened to define opportunities to improve patient access to personalized medicine through best practices, successful reimbursement models, high-quality economic evaluations, and strategic alignment. Session topics included health technology assessment, health economics, health policy, and value-based payment models and innovation.

\section{Introduction}

Health economics and economic evaluation aim to allocate the limited resources available in the most effective ways in various healthcare systems [1], not only to achieve optimal benefit for patients, but also to maintain the sustainability of national healthcare systems. As such, economic evaluation analyses are considered to be an integral part of the decision-making process as to whether a new intervention should or should not be adopted and incorporated in the

Sara L. Rogers

sara@stopadr.org

1 American Society of Pharmacovigilance, Houston, TX, USA

2 Department of Pharmacy, School of Health Sciences, University of Patras, Patras, Greece

3 Department of Pathology, College of Medicine \& Health Sciences, United Arab Emirates University, Al-Ain, UAE

4 Zayed Center of Health Sciences, United Arab Emirates University, Al-Ain, UAE

5 The Golden Helix Foundation, London, UK

6 Department of Pharmacy Services \& Intermountain Precision Genomics, Intermountain Healthcare Pharmacy Services, Taylorsville, UT, USA

7 Jerry H. Hodge School of Pharmacy, Texas Tech University Health Sciences Center, Dallas, TX, USA healthcare system [2, 3]. Also, it is of utmost importance that these innovative interventions are reimbursed by national healthcare systems. As such, and in order to make such informed decisions, it is critical that health policymakers are provided not only with the necessary evidence of clinical validity, but also with utility data associated with the genomic tests, as well as reliable evidence of economic benefit $[4,5]$. In other words, it is vital to provide reliable economic data demonstrating that reimbursing the cost of such genomic tests will not only improve patient quality of life but also reduce the costs of the overall national healthcare expenditure while increasing the efficiency of the public healthcare sector by guiding patients to personalized treatment recommendations [6].

A primary goal of the Golden Helix Foundation and Pharmacogenomics Access \& Reimbursement Coalition (PARC) is the exchange of ideas and experiences among stakeholders that share similar opportunities and challenges in pharmacogenomics. As such, the inaugural Pharmacogenomics Access \& Reimbursement Symposium (PARS) provided presentations by industry and thought leaders during key sessions including health technology assessment (HTA), Health Economics and Value-based Payment Strategies for Pharmacogenomics. Decision-makers from industry, government agencies, payer organizations, health systems and health policy organizations convened to define opportunities to improve patient access to precision medicine through best practices, successful reimbursement 
models, high-quality economic evaluations and strategic alignment.

The meeting was endorsed by the Golden Helix Foundation's Genomic Medicine Alliance (www. genomicmedicinealliance.org) and held a live webcasting that allowed virtual attendance. In the following pages, we provide an overview of the inaugural PARS, the description of the lecture content and an outline of the lively discussions held.

\section{Health technology assessment}

Finn Børlum Kristensen (Science \& Policy Consultancy) gave the opening presentation for the HTA session. He highlighted the urgent need for international development of methodology guidance for HTA and outcomes research and consensus on best practices in personalized medicine. In describing clinical implementation of pharmacogenomics in British Columbia, Canada, Stirling Bryan (BC Academic Health Science Network) advocated for pathway modeling to drive improvement in a clinical area. "Pathway modeling becomes the foundation of the HTA activity. It provides infrastructure by which we can robustly answer questions about the effectiveness and cost effectiveness of interventions in that pathway, drawing off data that has been published in clinical trials and other sources as way of synthesizing data while placing it into the jurisdictional context". He also encouraged resource stewardship and bringing stakeholders together to have open conversations about resource scarcity. "We have limited capacity to introduce new technology, so we want to use those resources well and drive as much improvement in care and health to the clinical population". April Zambelli -Weiner (TTi Health Research \& Economics) discussed the lack of alignment of evidence types and standards. "HTA is one of our key translational tools, but it is creating a bottleneck. Decades of research have identified many gene-drug pairs, a small portion of those have been approved by FDA for therapeutic management and only a very small percentage of patients have access". Dr. Zambelli-Weiner supported a shift in focus from randomized control trial study designs to real-world evidence generation strategies as well as health system and payer incentives for decreasing costs through more impactful HTA.

\section{Health economics}

Richard Willke (ISPOR) gave opening remarks during the Health Economics session, noting that personalized medicines have great potential to generate value in newer areas by reducing uncertainty, treating more severe diseases, increasing the probability of a cure, and other areas where standard cost-effectiveness analysis is a good tool but may not capture the full value. Marc Williams (Geisinger Health System) remarked that analyses performed from a societal perspective do not translate well to medical decisionmaking at the health system level. He discussed the possibility of a generic pharmacogenomic cost-effectiveness model to enable use of local input values and offer an efficient and timely value-based decision-making tool. "Implementing this approach demonstrates that costeffectiveness analyses can be rapidly performed without extensive training in decision modeling to provide useful evidence for decision-making and facilitate understanding about what conditions can meet cost-effectiveness thresholds". Kristine Ashcraft (Invitae) highlighted the need to store pharmacogenetic information discretely, reimburse ongoing clinical decision support in addition to the cost of the test and align reimbursement with evidence. She urged the United States Preventative Task Force (USPSTF) to consider recommending pharmacogenetics testing preemptively for high-risk patients.

\section{Health policy}

Frank Federico (Institute for Healthcare Improvement) offered opening remarks during the Health Policy session and discussed equity in health care, emphasizing the barriers to genomic services in relation to social determinants of health access. John Rother (National Coalition on Health Care) proposed the use of independent Comparative Effectiveness Research processes with arbitration as an approach to achieve innovation and price controls without heavy government regulation. He also advocated for systems that avoid shifting costs from one program to the next (i.e., cost-shifting). Joseph Antos (American Enterprise Institute) explained how pharmacogenomics testing could put a kink in the Pharmacy Benefits Manager (PBM) model since revenues are generated by creating formularies and making deals with drug manufacturing companies. "If it turns out that there is a sufficiently large number of patients with a particular genetic characteristic that goes against a particular drug on the formulary - how are they going to adjust their business practices to respond to this change in medical practices"? He recommended organizing a demonstration project with the Center for Medicare \& Medicaid Innovation (CMMI) as an approach to address formulary issues that might slow adoption of this technology and figure out what the business model is in the context of a healthcare system that relies on PBMs and private drug plans. "It is a difficult process, but I encourage the leaders in this sector to pursue it".

\section{Value-based payment models and innovation}

Rick Gundling (Healthcare Financial Management Association) explained the importance of building value-driving 
capabilities in health systems, noting that finance leaders are looking at the big picture in terms of how to deliver highvalue healthcare. Sara Teppema (Alta Advisers) explained the key building blocks for outcomes evaluation design. She suggested the possibility of a reduced fee-for-service model paired with savings accrual back to the healthcare provider over time as one approach to overcoming challenges associated with the short time period that payers typically evaluate interventions. The session closed with a robust panel discussion with Brandon Batiste (DC Connected Care Network), Gabriel Bien-Willner (MolDx; Palmetto GBA) and Pamela Pelizzari (Milliman). The discussion underscored the feasibility of a pharmacogenomics CMMI demonstration project, applications of coverage with evidence development and the role of pharmacists in valuebased payment initiatives.

Closing remarks at the symposium, by Benjamin Isgur $(\mathrm{PwC})$, reinforced the impetus to define the precision medicine landscape in the new health economy created by COVID-19. "Companies will need innovative financial models, robust data strategies and organizational changes to successfully navigate the changing global market for medicines and to balance product prices and value with affordability and patient access". The 2nd Annual Pharmacogenomics Access and Reimbursement Symposium will be held in fall 2021 in Washington, DC All are welcomed.

\section{Conference details}

The inaugural PARS was organized on October 8, 2020, at the National Academy of Sciences of the United States of America in Washington, DC The meeting was co-organized by the Golden Helix Foundation (www.goldenhelix.org) and the PARC (www.parcoalition.org), and the theme of the symposium was "Uniting Internationally to Advance Precision Medicine".

Acknowledgements This article was compiled by members of the Pharmacogenomics Access and Reimbursement Symposium 2020 organizing committee. The authors would like to acknowledge the symposium partners and supporters, including the Medical Device Innovation Consortium (MDIC), Medical Device Manufacturers Association (MDMA), Pharmacogenomics Research Network (PGRN), Society of Physician Entrepreneurs (SoPE).

\section{Compliance with ethical standards}

Conflict of interest GPP is Full Member and National Representative of the European Medicines Agency, Committee of Human Medicinal Products (CHMP) - Pharmacogenomics Working Party, Amsterdam, the Netherlands. BGB owns and operates Dynamic Life Sciences (DLS), a strategy and technology consulting firm.

Publisher's note Springer Nature remains neutral with regard to jurisdictional claims in published maps and institutional affiliations.

\section{References}

1. Williams A. Health economics: the cheerful face of a dismal science?. In: Health and economics. British Association for the Advancement of Science. London: Palgrave Macmillan; 1987.

2. McFarland A. Economic evaluation of interventions in health care. Nurs Stand. 2014;29:49-58.

3. Jonsson B. Ten arguments for a societal perspective in the economic evaluation of medical innovations. Eur $\mathrm{J}$ Health Econ. 2009;10:357-9.

4. Snyder SR, Mitropoulou C, Patrinos GP, Williams MS. Economic evaluation of pharmacogenomics: a value-based approach to pragmatic decision making in the face of complexity. Public Health Genom. 2014;17:256-64.

5. Patrinos GP, Mitropoulou C. Measuring the value of pharmacogenomics evidence. Clin Pharmacol Ther. 2017;102:739-41.

6. Vozikis A, Cooper N, Mitropoulou C, Kambouris E. Test pricing and reimbursement in genomic medicine: Towards a general strategy. Public Health Genom. 2016;19:352-63. 